\title{
Longitudinal Component Properties of Circularly Polarized Terahertz Vortex Beams
}

\author{
Miao Wang, Xinke Wang *, Peng Han, Wenfeng Sun, Shengfei Feng, Jiasheng Ye and \\ Yan Zhang
}

Department of Physics, Beijing Key Lab for Metamaterials and Devices, Capital Normal University, Beijing, China

A circularly polarized vortex beam possesses similar focusing properties as a radially polarized beam. This type of beam is highly valuable for developing optical manufacturing technology, microscopy, and particle manipulation. In this work, a lefthand circularly polarized terahertz (THz) vortex beam (CPTVB) is generated by utilizing a $\mathrm{THz}$ quarter wave plate and a spiral phase plate. Focusing properties of its longitudinal

OPEN ACCESS

Edited by:

Yiqi Zhang,

Xi'an Jiaotong University, China

Reviewed by:

Dong Mao,

Northwestern Polytechnical

University, China

Tongyi Zhang,

Xian Institute of Optics and Precision Mechanics (CAS), China

Zuanming Jin,

University of Shanghai for Science and

Technology, China

*Correspondence: Xinke Wang wxk82721@cnu.edu.cn

Specialty section:

This article was submitted to

Optics and Photonics,

a section of the journal

Frontiers in Physics

Received: 05 July 2021 Accepted: 18 August 2021 Published: 31 August 2021

Citation:

Wang $M$, Wang $X$, Han $P$, Sun $W$,

Feng S, Ye J and Zhang Y (2021)

Longitudinal Component Properties of

Circularly Polarized Terahertz

Vortex Beams.

Front. Phys. 9:736831.

doi: 10.3389/fphy.2021.736831 component $E_{z}$ are detailedly discussed on the simulation and experiment. With reducing the $\mathrm{F}$-number of the $\mathrm{THz}$ beam and comparing with a transverse component $E_{x}$ of a general circularly polarized $\mathrm{THz}$ beam, the simulation results show that the focal spot size and intensity of its $E_{z}$ component can reach 87 and $50 \%$ of $E_{x}$ under a same focusing condition. In addition, the experimental results still demonstrate that the left-hand CPTVB can always maintain fine $E_{z}$ focusing properties in a broad bandwidth, which manifest the feasibility of this class of $\mathrm{THz}$ beams.

Keywords: terahertz, circularly polarized vortex beam, longitudinal component, focusing properties, dispersive characteristics

\section{INTRODUCTION}

As the maturation of terahertz $(\mathrm{THz})$ technology, this kinds of far-infrared sensing and imaging methods have gradually presented powerful application values in many research and industrial fields [1, 2]. Recently, investigations and applications of $\mathrm{THz}$ special beams have obviously become a hot spot and attracted growing attention. Taking advantages of their distinctive diffraction characteristics, all kinds of special beams have been successfully applied in $\mathrm{THz}$ imaging [3], $\mathrm{THz}$ communications [4], electron acceleration [5], and so on. In 2006, Zhan Q. W. theoretically proposed that the longitudinal electric field component of a circularly polarized vortex beam is analogous to that of a radially polarized beam, which possesses a sharper focal spot [6]. In 2016, our report experimentally verified the properties of this kind of optical beam in the THz waveband and pointed out its application prospects in THz microscopy and particle acceleration [7]. In 2017, Minasyan A. et. al designed a space-variant birefringent slab to achieve a circularly polarized THz vortex beam (CPTVB) [8]. In 2019, Sirenko A. A. et. al demonstrated that modified broadband circularly polarized $\mathrm{THz}$ vortices can be utilized as a spectroscopic probe of magnetism [9]. In 2021, Sobhani $\mathrm{H}$. theoretically discussed the creation of a $\mathrm{THz}$ pulse carrying orbital angular momentum via beating twisted laser pulses in the plasma [10]. Apparently, the generation methods and characteristics of CPTVBs have gained more and more attention. Compared to a radially polarized THz beam, a CPTVB can be more easily produced and modulated by using a THz quarter wave plate (TQWP) and a spiral phase plate (SPP). Therefore, a further study on longitudinal component features of a CPTVB is valuable for improving the performances of current $\mathrm{THz}$ systems. 


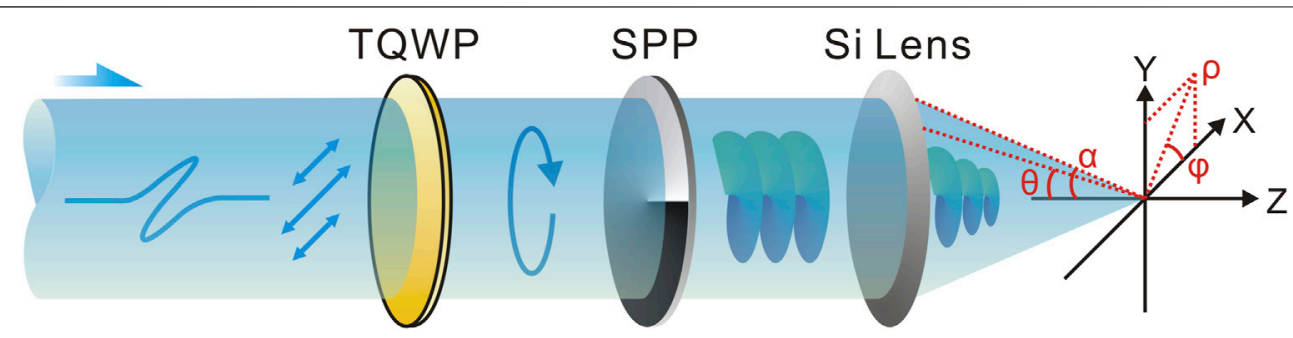

FIGURE 1 | Schematic diagram of a circularly polarized THz vortex beam (CPTVB). TQWP: THz quarter wave plate; SPP: spiral phase plate.

In this paper, we compared focusing properties of the longitudinal component of a CPTVB and the transverse component of a general circularly polarized $\mathrm{THz}$ beam on the simulation and experiment. Under different focusing conditions, evolutions of their focal spots are presented and analyzed. Besides, the dispersive characteristics of the longitudinal component of a CPTVB produced by using a TQWP and a SPP are also observed and discussed.

\section{SIMULATION}

Firstly, we present and analyze the field distributions of CPTVBs on the simulation. Figure 1 gives the schematic diagram of a CPTVB generated by a TQWP and a SPP. The incident THz wave with a $\mathrm{x}$-linear polarization illuminates a TQWP to possess a circular polarization. Then, the circularly polarized $\mathrm{THz}$ beam carries a spiral wave front after passing through a SPP. To generate a strong longitudinal electric field component, a high resistivity silicon (Si) lens is adopted to focus the THz beam. Here, a modified RichardsWolf integration algorithm is utilized to simulate vector $\mathrm{THz}$ field components [11]. In the high-aperture aplanatic focusing system, the focal spot is located at a sufficient distance away from the aperture. Then, vector components of a $\mathrm{THz}$ vortex beam in a homogeneous dielectric medium near the focus can be written as

$$
E(\rho, \varphi, z)=-j k f \int_{0}^{\alpha} Q(\rho, \varphi, \theta) q(\theta) d \theta,
$$

where $(\rho, \varphi, z)$ is the cylindrical coordinate on an observation plane, $k=2 \pi / \lambda$ is the wave number in the vacuum, $\lambda$ is the wavelength of the incident THz beam, $f$ is the focal length of the Si lens, $\theta$ is the angle between the converging THz beam and the $z$ axis, $\alpha$ is the maximum value of $\theta . q(\theta)$ can be described as $q(\theta)=\sqrt{\cos \theta} \sin \theta \exp (j k z \cos \theta)$, which is related to the pupil apodization function of the focusing system. The expression of $\mathrm{Q}(\rho, \varphi, \theta)$ depends on the incident $\mathrm{THz}$ polarization. When the incident $\mathrm{THz}$ field possesses a circular polarization, $\mathrm{Q}(\rho, \varphi, \theta)$ can be written as

$$
\begin{aligned}
& \mathrm{Q}(\rho, \varphi, \theta)=\frac{1}{\sqrt{2}} j^{m} \exp (j m \varphi) \\
& \times\left[\begin{array}{l}
J_{m}(t)+\frac{1}{2}\left[J_{m}(t)+B_{m}^{E E}(t, \varphi)\right](\cos \theta-1) \\
\operatorname{sgn}(p) j\left\{J_{m}(t)+\frac{1}{2}\left[J_{m}(t)-B_{m}^{E E}(t, \varphi)\right](\cos \theta-1)\right\} \\
-B_{m}^{E}(t, \varphi) \sin \theta
\end{array}\right]
\end{aligned}
$$

where $m$ is the topological charge of the SPP, sgn $(p)$ is the sign of the input polarization, $J_{m}(t)$ is the Bessel function of the first kind and $t=k \rho \sin \theta$. In addition,

$$
\begin{gathered}
B_{m}^{E E}(t, \varphi)=-\exp (\operatorname{sgn}(p) j 2 \varphi) J_{m+\operatorname{sgn}(p) 2}(t), \\
B_{m}^{E}(t, \varphi)=\operatorname{sgn}(p) j \exp (\operatorname{sgn}(p) j \varphi) J_{m+\operatorname{sgn}(p) 1}(t) .
\end{gathered}
$$

In the initial simulation, these parameters are set as $\lambda=$ $400 \mu \mathrm{m}$ (corresponding to $0.75 \mathrm{THz}$ ), $f=20 \mathrm{~mm}, \alpha=26.56^{\circ}$, $\operatorname{sgn}(p)=1$ (corresponding to a left-hand circular polarization). By using Eqs. 1-4, the polarization components $E_{x}$ and $E_{z}$ of a left-hand CPTVB with $m=0$ are simulated and presented on the focal plane, as shown in Figures 2A,B and Figures 2E,F. Figures $2 \mathrm{~A}, \mathbf{B}$ give the amplitude and phase distributions of $E_{x}$. Figures 2E,F show the amplitude and phase patterns of $E_{z}$. When $m=0$, the CPTVB is just a general circularly polarized THz beam. Therefore, $E_{x}$ shows a typical focal spot and its phase nearly exhibits a flat plane near the focal spot. Meanwhile, the $E_{z}$ component presents a vortex pattern, including a doughnutshaped amplitude and a spiral phase in a clockwise direction. These phenomena can be easily explained. With a circular polarization, longitudinal components $E_{z}$ can be simultaneously generated on both X-Z and Y-Z planes and a $\pi / 2$ phase difference exists between them, so the interference between them results in the $E_{z}$ morphology of the CPTVB with $m=0$. When the topological charge of the SPP is set as $m=-1$, a spiral phase modulation is introduced into the $\mathrm{THz}$ beam. In this case, the $E_{x}$ and $E_{z}$ components are simulated and presented in Figures 2C,D and Figures 2G,H. It can be seen that $E_{x}$ shows a ring-shaped amplitude and a spiral phase in a counterclockwise direction, as shown in Figures 2C,D. In Figures $2 \mathrm{G}, \mathbf{H}$ the $E_{z}$ amplitude exhibits a main focal spot as well as some weak annular side-lobes and the $E_{z}$ phase is composed of a series of concentric rings. On the interfaces between adjacent ring-shaped phases, there are always a $\pi$ phase jump. To understand the features of the $E_{z}$ component, a normalized left-hand circularly polarized $\mathrm{THz}$ beam can be expressed as [6].

$$
\mathbf{E}_{L H C}=\exp (j \varphi)\left(\mathbf{e}_{r}+j \mathbf{e}_{\varphi}\right) / \sqrt{2},
$$

where $\mathbf{e}_{r}$ and $\mathbf{e}_{\varphi}$ are the unit vectors on radial and azimuthal directions. When a spiral phase modulation with $m=-1$ is loaded on the $\mathrm{THz}$ beam, the original spiral phase term $\exp (j \varphi)$ is eliminated and the $\mathrm{THz}$ field can be expressed as 


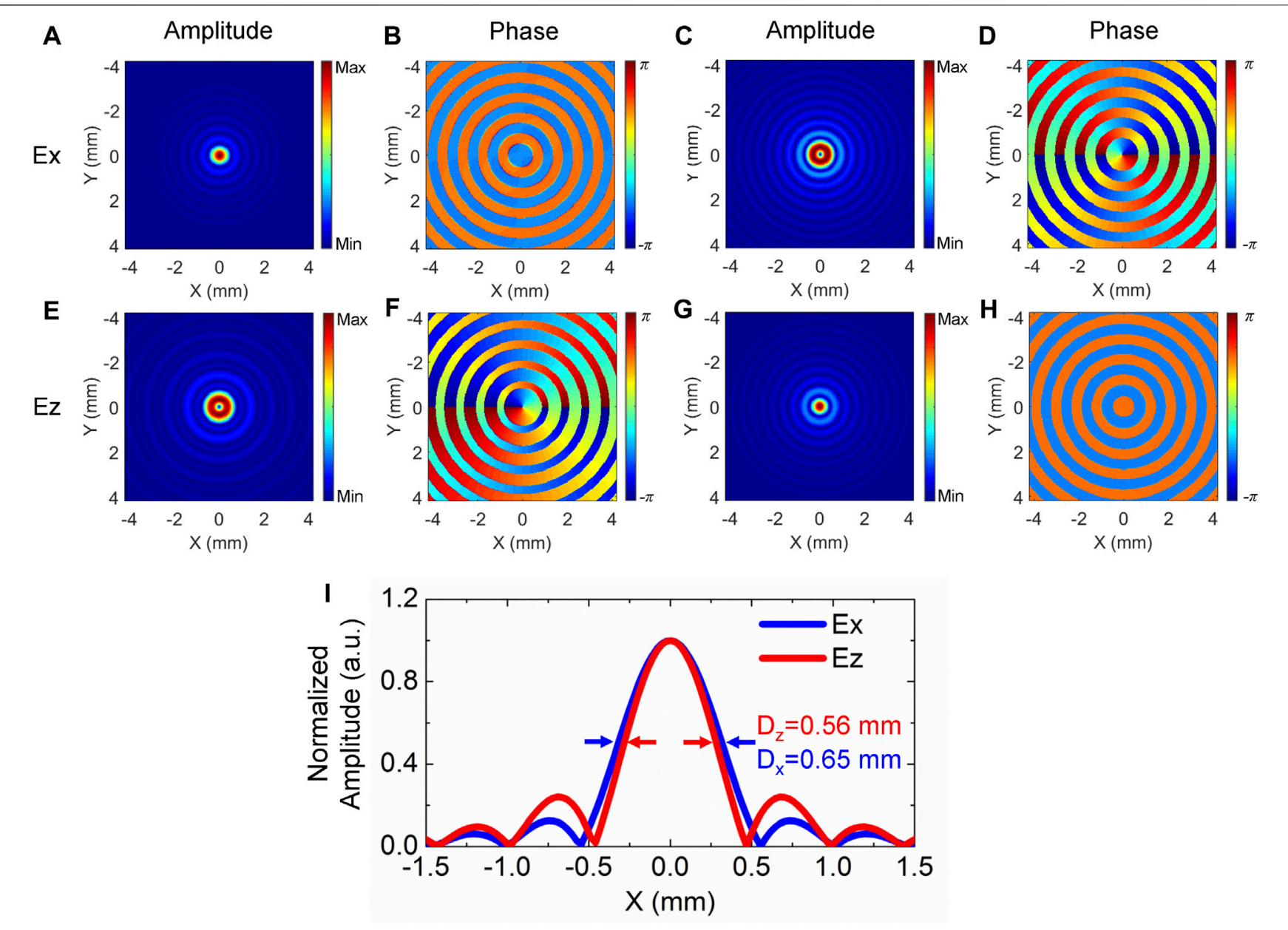

FIGURE 2 | (A,E) Amplitude and (B,F) phase patterns of $E_{x}$ and $E_{z}$ for a left-hand CPTVB with $m=0$. (C,G) Amplitude and (D,H) phase distributions of $E_{x}$ and $E_{z}$ for a left-hand CPTVB with $m=-1$. (I) Normalized amplitude profile curves of $E_{x}$ with $m=0$ and $E_{z}$ with $m=-1$. These curves are extracted from (A) and (G), respectively.

$$
\mathbf{E}=\left(\mathbf{e}_{r}+j \mathbf{e}_{\varphi}\right) / \sqrt{2} .
$$

Then, the $\mathrm{THz}$ beam can be considered as a linear superposition of the $\mathbf{e}_{r}$ and $\mathbf{e}_{\varphi}$ components. When the $\mathrm{THz}$ beam is focused, the $\mathbf{e}_{\varphi}$ component cannot induce a longitudinal electric field component, so the formation of $E_{z}$ completely arises from the contribution of the $\mathbf{e}_{r}$ component [12]. Therefore, the $E_{z}$ distribution features of the left-hand CPTVB with $m=-1$ are very similar to the longitudinal component of a radially polarized $\mathrm{THz}$ beam. These simulation results are very consistent with our previous report [7].

In addition, it should be noted that a right-hand circularly polarized $\mathrm{THz}$ beam can be also used to generate a sharp real focus of $E_{z}$ in a similar manner. When a spiral phase modulation with $m=1$ is loaded on a THz beam with a right-hand circular polarization, the constructive interference of $E_{z}$ is fulfilled on the optical axis and a focal spot of $E_{z}$ is formed. However, $E_{z}$ suffers from destructive interference when a spiral phase modulation with $m=-1$ is loaded on a right-hand circularly polarized $\mathrm{THz}$ beam. In that case, the $E_{z}$ component will present a vortex pattern with a topological charge of -2 . The related discussions have been reported in our previous work [7].

The most important property of a converging radially polarized beam is that the focal spot size of its $E_{z}$ component is smaller than that of the $E_{x}$ component of a general focused beam with a same F-number, which is very valuable for optical microscopy [13] and particle acceleration [5]. Herein, we compare the focal spot sizes of $E_{z}$ with $m=-1$ and $E_{x}$ with $m=0$ for left-hand CPTVBs. The amplitude profiles of $E_{z}$ with $m=-1$ and $E_{x}$ with $m=0$ are separately extracted along the $x$ axis from Figures 2A,G. Their normalized curves are plotted and compared, as shown in Figure 2I. The full width half maximum (FWHM) $D_{z}$ and $D_{x}$ of $E_{z}$ and $E_{x}$ are marked by red and blue arrows, which are 0.56 and $0.65 \mathrm{~mm}$, respectively. It clearly manifests that a CPTVB can be utilized to form a smaller focal spot.

To further analyze $E_{z}$ properties of a CPTVB, the focal length $f$ is varied from 10 to $80 \mathrm{~mm}$ and other parameters are fixed in the simulation. The amplitude patterns of $E_{x}$ with $m=0$ and $E_{z}$ with $m=-1$ of left-hand CPTVBs are simulated and their amplitude profiles are extracted along the $x$ axis. Figures 3A,B exhibit the 

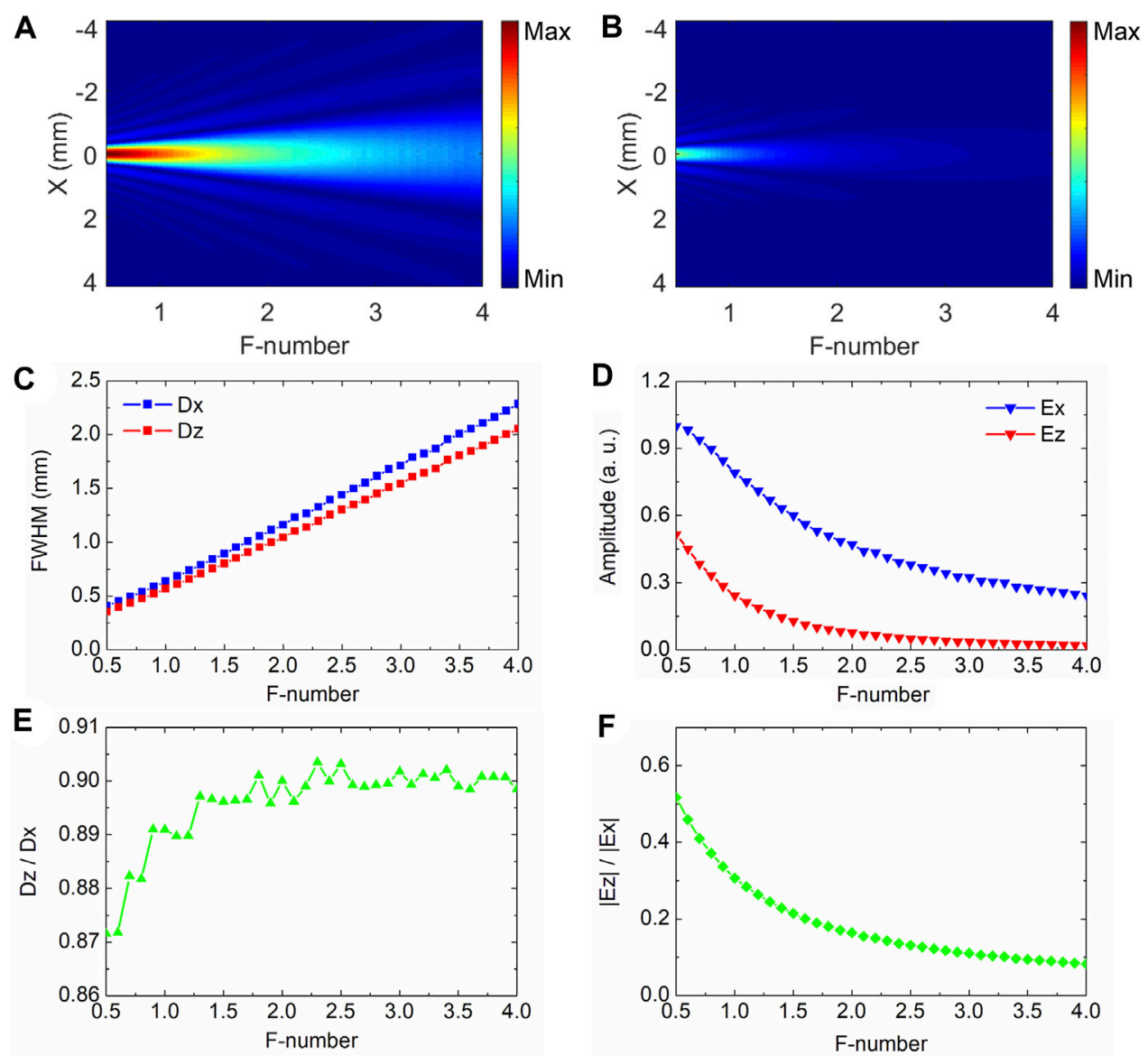

FIGURE 3 | Variations of amplitude profiles with different F-numbers for (A) $E_{x}$ with $m=0$ and (B) $E_{z}$ with $m=-1$ of left-hand CPTVBs. (C) and (E) give the variation tendencies of the FWHMs $D_{x}$ and $D_{z}$ of $E_{x}$ and $E_{z}$ and their ratio $D_{z} / D_{x}$ with varying the F-number. (D) and (F) exhibit the evolution trends of $E_{x}$ and $E_{z}$ amplitude peaks and their ratio $\left|E_{z}\right|\left|E_{x}\right|$ with adjusting the F-number.

amplitude profile distributions of $E_{x}$ and $E_{z}$ along different F-numbers. Obviously, their common points are that the focal spot sizes of $E_{x}$ and $E_{z}$ monotonically enlarge and their intensities progressively attenuate with increasing the F-number. To more intuitively observe the variation tendencies of their focal spots, the FWHMs $D_{x}$ and $D_{z}$ of $E_{x}$ and $E_{z}$ are extracted and plotted in Figure 3C. $D_{x}$ and $D_{z}$ vary from $0.40 \mathrm{~mm}$ and $0.35 \mathrm{~mm}$ to $2.28 \mathrm{~mm}$ and $2.05 \mathrm{~mm}$ with adjusting the F-number from 0.5 to 4 . Meanwhile, it is apparent that $D_{z}$ is always smaller than $D_{x}$ with different F-numbers. In addition, the ratio of $D_{z}$ to $D_{x}$ is also calculated and exhibited in Figure 3E, which alters from 0.87 to 0.90 with changing the F-number from 0.5 to 4 . It can be found that the ratio almost remains unchanged with decreasing the F-number from 4 to 1.5 and it sharply reduces when the F-number is less than 1.5. Besides, intensities of $E_{x}$ and $E_{z}$ with different F-numbers are also compared and analyzed. Figure 3D gives the variations of the $E_{x}$ and $E_{z}$ amplitude peaks at $x=0$ with changing the F-number. It should be noted that both $E_{x}$ and $E_{z}$ amplitudes are normalized to the $E_{x}$ amplitude peak. It can be observed that both $E_{x}$ and $E_{z}$ amplitudes monotonically enhance with decreasing the F-number and the evolution trend of $E_{z}$ is more pronounced when the F-number is less than 1.5. The ratio $\left|E_{z}\right| /\left|E_{x}\right|$ of the $E_{x}$ and $E_{z}$ amplitudes is also calculated and shown in Figure 3F, which shows that the proportion of $E_{z}$ is more and more significant with reducing the F-number and can approach $50 \%$ with the F-number of 0.5 . According to these simulation results, it indicates that a focusing condition with a smaller F-number is essential for acquiring a $E_{z}$ focal spot with a smaller size and a higher intensity.

\section{EXPERIMENT}

On the experiment, CPTVBs are also achieved and analyzed. A $\mathrm{THz}$ focal-plane imaging system is applied to characterize the features of CPTVBs, as shown in Figure 4. A Spectra-Physics femtosecond laser amplifier ( $800 \mathrm{~nm}$ central wavelength, $35 \mathrm{fs}$ pulse duration, $1 \mathrm{~W}$ average power, and $1 \mathrm{kHz}$ repetition ratio) is used as the light source. The laser pulse is divided into the pump and probe beams by a half wave plate (HWP) and a polarization beam splitter (PBS) for exciting and detecting the $\mathrm{THz}$ wave. After passing through a motorized linear stage, the pump beam with a $990 \mathrm{~mW}$ average power is expanded by a concave lens L1 with a $50 \mathrm{~mm}$ focal length and is guided to illuminate a $<110>$ 


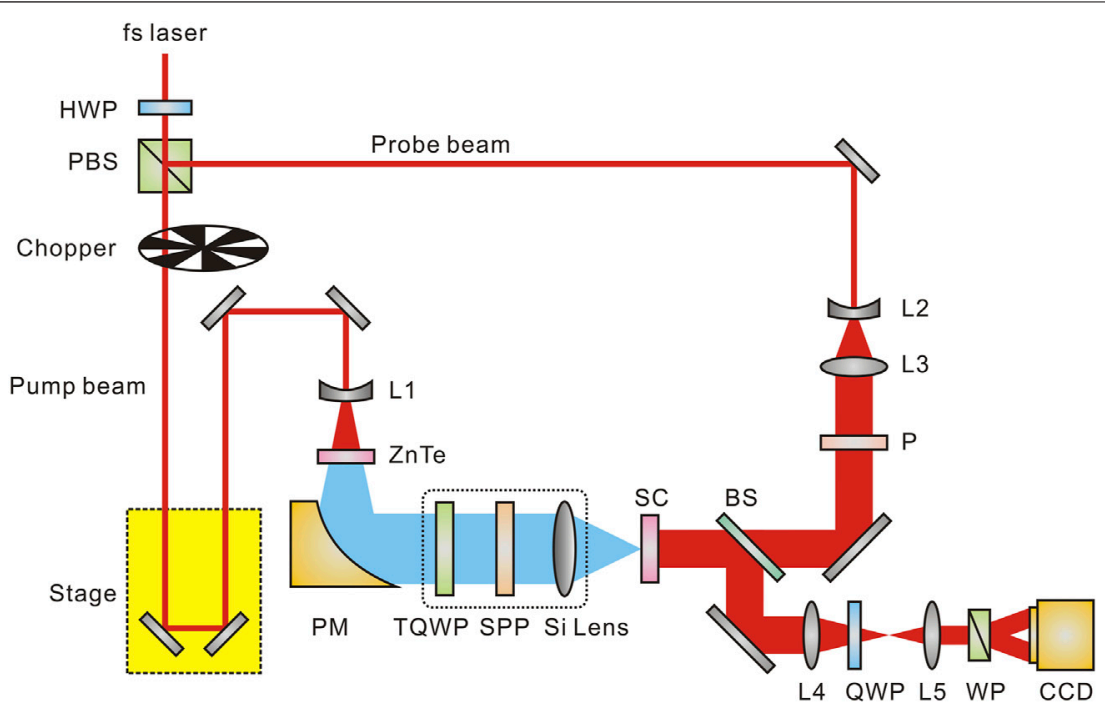

FIGURE 4 | Schematic diagram of a THz focal-plane imaging system.

$\mathrm{ZnTe}$ crystal with a $2 \mathrm{~mm}$ thickness. Then, the $\mathrm{THz}$ radiation with a linear polarization is generated by the optical rectification effect [14]. An axis-off parabolic mirror (PM) with a $100 \mathrm{~mm}$ focal length is utilized to collimate the $\mathrm{THz}$ beam. Herein, the diameter of the $\mathrm{THz}$ beam is approximately $20 \mathrm{~mm}$. After successively transmitting through a TQWP, a SPP and a Si lens, a converging left-hand CPTVB is formed and is incident into the sensor crystal (SC). On the path of the probe beam, the laser pulse with a $10 \mathrm{~mW}$ average power is sequentially expanded and collimated by concave and convex lenses (L2 and L3) with focal lengths of 50 and $150 \mathrm{~mm}$. The diameter of the probe beam roughly reaches $30 \mathrm{~mm}$. A polarizer $(\mathrm{P})$ is used to ensure the probe polarization and the probe beam is reflected onto the SC. In the SC, the two-dimensional $\mathrm{THz}$ information is modulated on the probe polarization by the linear electro-optic effect [15]. The probe beam carrying the $\mathrm{THz}$ information is reflected by the SC and a 50/50 non-polarizing beam splitter in sequence and is guided into the imaging module of the system, which is constituted of a lens group (L4 and L5), a quarter wave plate (QWP), a Wollaston prism (WP), and a CCD camera with a $4 \mathrm{~Hz}$ frame rate. The imaging module is applied to capture the image of the probe beam on the SC. A mechanical chopper is mounted in the pump beam to modulate the output frequency of the $\mathrm{THz}$ pulse and is synchronously controlled with the CCD camera. Dynamics subtraction and balanced electro-optic detection methods are adopted to remove the background intensity of the probe beam $[16,17]$ and a two-dimensional $\mathrm{THz}$ image is accurately extracted. By continuously adjusting the time delay between the pump and probe beams, a series of $\mathrm{THz}$ temporal images are measured and the Fourier transformation is operated on each pixel to acquire the $\mathrm{THz}$ spectral information. To suppress the background noise of the system, 25 frames are averaged at each temporal scan point. In this system, the effective imaging area is $12 \mathrm{~mm} \times 12 \mathrm{~mm}$ and the size of a pixel is $57 \mu \mathrm{m}$.
To characterize the different polarization components of the $\mathrm{THz}$ beam, the SCs with various crystalline orientations are carefully selected. In the measurement, the probe polarization is always fixed as the horizontal direction. $\mathrm{A}<110>\mathrm{ZnTe}$ with a $1 \mathrm{~mm}$ thickness is chosen to measure the transverse electric field $E_{x}$ of the $\mathrm{THz}$ beam. To maximize the detection efficiency, the angle between the $<001>$ direction of the crystal and the probe polarization is set as $0^{\circ} . \mathrm{A}<100>\mathrm{ZnTe}$ with a $1 \mathrm{~mm}$ thickness is selected to acquire the longitudinal electric field $E_{z}$ of the $\mathrm{THz}$ beam. The $\langle 010\rangle$ direction of the crystal is tuned to $45^{\circ}$ with respect to the probe polarization to optimize the detection efficiency [7].

To achieve a left-hand circularly polarized $\mathrm{THz}$ beam, a quartz TQWP (TYDEX Company, Russia) with a $400 \mu \mathrm{m}$ central wavelength is applied. A Teflon SPP with a topological charge of -1 and a $400 \mu \mathrm{m}$ central wavelength is used to impart a spiral phase modulation on the $\mathrm{THz}$ beam. Three Si lenses with focal lengths of $20 \mathrm{~mm}, 30 \mathrm{~mm}, 50 \mathrm{~mm}$ are separately picked up to produce the converging CPTVBs for comparing the features of $E_{z}$ with different focusing conditions. On the focal plane, the $E_{x}$ and $E_{z}$ components of the left-hand CPTVBs are measured and analyzed by using the imaging system.

Figure 5 exhibits the comparison of the $E_{x}$ and $E_{z}$ components under different focusing conditions. Figures 5A,D,G give the amplitude distributions of the focal spots with $f=20 \mathrm{~mm}, 30 \mathrm{~mm}$, $50 \mathrm{~mm}$ at $0.75 \mathrm{THz}$ for $E_{x}$ with $m=0$ of left-hand CPTVBs. Figures 5B,E,H show the amplitude patterns of $E_{z}$ with three different focal lengths at $0.75 \mathrm{THz}$ on the focal plane for left-hand CPTVBs with $m=-1$. Obviously, the focal spot sizes of $E_{x}$ and $E_{z}$ are gradually magnified with increasing $f$. For clarity, their corresponding amplitude profile curves are extracted along the $x$ axis. The normalized curves of $E_{x}$ and $E_{z}$ with $f=20 \mathrm{~mm}, 30 \mathrm{~mm}, 50 \mathrm{~mm}$ are plotted and compared, as shown in Figures 5C,F,I. When the focal length of the Si lens is $20 \mathrm{~mm}$, the FWHMs $D_{x}$ and $D_{z}$ of $E_{x}$ and $E_{z}$ are 0.87 and $0.61 \mathrm{~mm}$, respectively. When $f$ is adjusted as $30 \mathrm{~mm}$, 

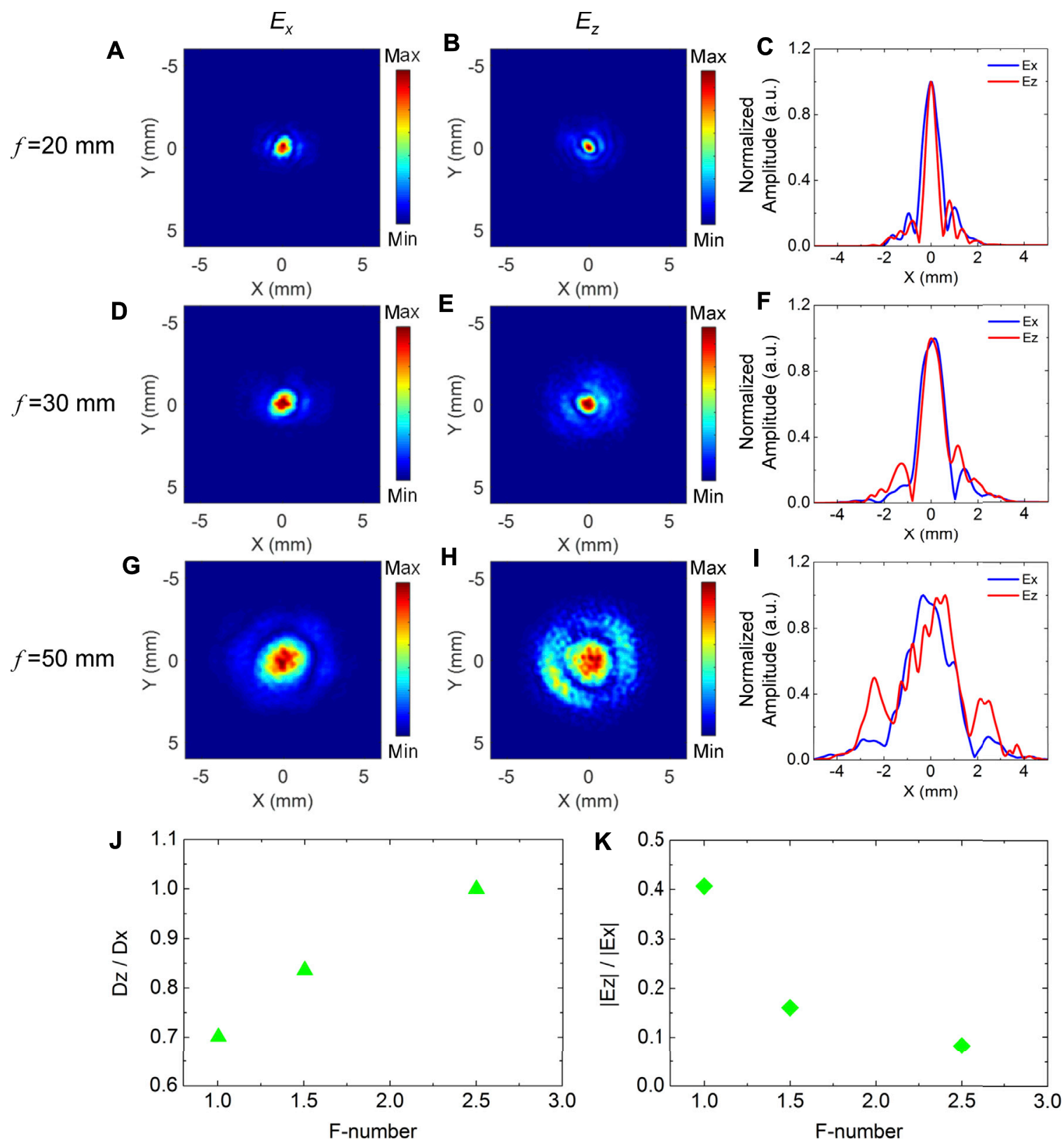

FIGURE 5 | Comparison of the transverse and longitudinal components under different focusing conditions. (A), (D), (G) and (B), (E), (H) separately present the amplitude distributions of the focal spots with focal lengths of $20 \mathrm{~mm}, 30 \mathrm{~mm}, 50 \mathrm{~mm}$ at $0.75 \mathrm{THz}$ for $E_{x}$ with $m=0$ and $E_{z}$ with $m=-1$ of left-hand CPTVBs. (C), (F), (I) give their corresponding normalized amplitude profile curves along the $x$ axis $(\mathbf{J})$ and $(\mathbf{K})$ show the ratios of $D_{z} / D_{x}$ and $\left|E_{z}\right| /\left|E_{x}\right|$ with varying the $F-n u m b e r$, respectively.

$D_{x}$ and $D_{z}$ are 1.22 and $1.02 \mathrm{~mm}$. When $f$ is varied as $50 \mathrm{~mm}$, both $D_{x}$ and $D_{z}$ are almost equal to $2.32 \mathrm{~mm}$. Simultaneously, the sidelobes of $E_{z}$ becomes more striking. Experimental results are mainly consistent with the simulation. When a CPTVB is more tightly focused, the $E_{z}$ component with a smaller focal spot is formed. In addition, the focal spot size of $E_{z}$ is always less than that of $E_{x}$. Some slight deviations between the experimental and simulation results are mainly attributed to the integral effect of the $1 \mathrm{~mm}$-thick SCs [18] and other measurement errors. Besides, the ratios of $D_{z} / D_{x}$ and $\left|E_{z}\right| /\left|E_{x}\right|$ are also calculated and exhibited in Figures 5J,K, which presents similar tendencies as the simulation results.

Moreover, the dispersive characteristics of the focal spots with different focal lengths are also checked for $E_{z}$ with $m=-1$ of lefthand CPTVBs. From the measurement results of $E_{z}$, each spectral amplitude profile curves are extracted along the $x$ axis. Figures 6A-C present the amplitude profile distributions of $E_{z}$ with $f=20 \mathrm{~mm}, 30 \mathrm{~mm}$, and $50 \mathrm{~mm}$ along different frequencies. In Figures $\mathbf{6 A}, \mathbf{B}$, it can be seen that $E_{z}$ always possesses a clear real focus from $0.5 \mathrm{THz}$ to $1.0 \mathrm{THz}$. With $f=$ 

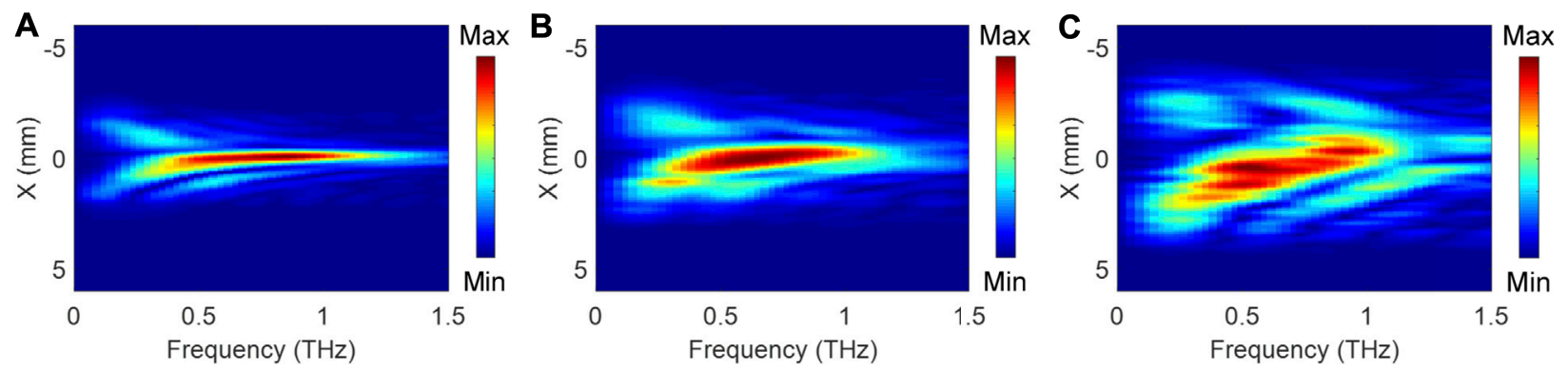

FIGURE 6 | Dispersive characteristics of the focal spots with $f=20 \mathrm{~mm}$ (A), $30 \mathrm{~mm}$ (B), and $50 \mathrm{~mm}$ (C) for the $E_{z}$ components with $m=-1$ of left-hand CPTVBs.

$50 \mathrm{~mm}$, the focal spot gets blurred at frequencies away from $0.75 \mathrm{THz}$ due to the weaker $E_{z}$ component, as shown in Figure 6C. It manifests that a CPTVB can generate a fine converging $E_{z}$ component with a broad bandwidth, although the modulation effects of the TQWP and SPP are the most perfect at the central wavelength.

\section{CONCLUSION}

In conclusion, the focusing properties of the $E_{z}$ component are analyzed in detail for a converging CPTVB. The simulation and experimental results show that a CPTVB can form a real $E_{z}$ focus when its topological charge is carefully adjusted. With reducing the F-number, the $E_{z}$ component can get a sharper focal spot and a higher intensity. Particularly, the focal spot size of $E_{z}$ is always less than that of the $E_{x}$ component of a general circularly polarized $\mathrm{THz}$ beam with the same F-number. In addition, $E_{z}$ can remain a fine focusing effect in a broad bandwidth for a CPTVB generated by a TQWP and a SPP. This work provides an effective avenue to produce a longitudinal $\mathrm{THz}$ polarization component with a smaller size and a stronger intensity. It can be expected that this class of CPTVBs will exhibit important application values for improving current $\mathrm{THz}$ inspection systems.

\section{REFERENCES}

1. Tonouchi M. Cutting-Edge Terahertz Technology. Nat Photon (2007) 1: 97-105. doi:10.1038/nphoton.2007.3

2. Guerboukha H, Nallappan K, and Skorobogatiy M. Toward Real-Time Terahertz Imaging. Adv Opt Photon (2018) 10:843-938. doi:10.1364/ AOP.10.000843

3. Bitman A, Moshe I, and Zalevsky Z. Improving Depth-Of Field in Broadband $\mathrm{THz}$ Beams Using Nondiffractive Bessel Beams. Opt Lett (2012) 37:4164-6. doi:10.1364/OL.37.004164

4. Hui X, Zheng S, Chen Y, Hu Y, Jin X, Chi H, et al. Multiplexed Millimeter Wave Communication with Dual Orbital Angular Momentum (OAM) Mode Antennas. Sci Rep (2015) 5:10148. doi:10.1038/srep10148

5. Nanni EA, Huang WR, Hong K-H, Ravi K, Fallahi A, Moriena G, et al. Terahertz-Driven Linear Electron Acceleration. Nat Commun (2015) 6:8486. doi:10.1038/ncomms9486

6. Zhan Q. Properties of Circularly Polarized Vortex Beams. Opt Lett (2006) 31: 867-9. doi:10.1364/OL.31.000867

\section{DATA AVAILABILITY STATEMENT}

The original contributions presented in the study are included in the article/Supplementary Material, further inquiries can be directed to the corresponding author.

\section{AUTHOR CONTRIBUTIONS}

MW performed the research and wrote the paper. XW and YZ proposed the concept. $\mathrm{PH}, \mathrm{WS}, \mathrm{SF}$, and JY supervised the project. All authors discussed the results and co-wrote the article.

\section{FUNDING}

This research was supported by the National Natural Science Foundation of China (61735002, 11774243, 11774246, and 11404224), Youth Innovative Research Team of Capital Normal University (008/20530290053, 008/19530050146, 008/ 18530500155), Connotative Development Foundation for Distinguished Young Talents in Capital Normal University (2055105), and Capacity Building for Sci-Tech InnovationFundamental Scientific Research Funds (008/20530290072, 008/19530050180, 025185305000/142).

7. Wang X, Shi J, Sun W, Feng S, Han P, Ye J, et al. Longitudinal Field Characterization of Converging Terahertz Vortices with Linear and Circular Polarizations. Opt Express (2016) 24:7178-90. doi:10.1364/ OE.24.007178

8. Minasyan A, Trovato C, Degert J, Freysz E, Brasselet E, and Abraham E. Geometric Phase Shaping of Terahertz Vortex Beams. Opt Lett (2017) 42:41-4 doi:10.1364/OL.42.000041

9. Sirenko AA, Marsik P, Bernhard C, Stanislavchuk TN, Kiryukhin V, and Cheong S-W. Terahertz Vortex Beam as a Spectroscopic Probe of Magnetic Excitations. Phys Rev Lett (2019) 122:237401. doi:10.1103/ PhysRevLett.122.237401

10. Sobhani H. Creation of Tunable Longitudinally Polarized Terahertz Pulse Carrying Orbital Angular Momentum. Phys Lett A (2021) 387:127011. doi:10.1016/j.physleta.2020.127011

11. Khonina SN, Kazanskiy NL, and Volotovsky SG. Vortex Phase Transmission Function as a Factor to Reduce the Focal Spot of High-Aperture Focusing System. J Mod Opt (2011) 58:748-60. doi:10.1080/09500340.2011.568710

12. Youngworth KS, and Brown TG. Focusing of High Numerical Aperture Cylindrical-Vector Beams. Opt Express (2000) 7:77-87. doi:10.1364/OE.7.000077 
13. Huse N, Schönle A, and Hell SW. Z-Polarized Confocal Microscopy. J Biomed Opt (2001) 6:480-4. doi:10.1117/1.1417974

14. Löffler T, Hahn T, Thomson M, Jacob F, and Roskos HG. Large-Area ElectroOptic ZnTe Terahertz Emitters. Opt Express (2005) 13:5353-62. doi:10.1364/ OPEX.13.005353

15. Wu Q, Litz M, and Zhang XC. Broadband Detection Capability of ZnTe Electro-Optic Field Detectorsfield Detectors. Appl Phys Lett (1996) 68:2924-6. doi:10.1063/1.116356

16. Jiang Z, Xu XG, and Zhang X-C. Improvement of Terahertz Imaging with a Dynamic Subtraction Technique. Appl Opt (2000) 39:2982-7. doi:10.1364/ AO.39.002982

17. Wang X, Cui Y, Sun W, Ye J, and Zhang Y. Terahertz Real-Time Imaging with Balanced Electro-Optic Detection. Opt Commun (2010) 283:4626-32. doi:10.1016/j.optcom.2010.07.010

18. Wang S, Zhao F, Wang X, Qu S, and Zhang Y. Comprehensive Imaging of Terahertz Surface Plasmon Polaritons. Opt Express (2014) 22:16916-24. doi:10.1364/OE.22.016916
Conflict of Interest: The authors declare that the research was conducted in the absence of any commercial or financial relationships that could be construed as a potential conflict of interest.

Publisher's Note: All claims expressed in this article are solely those of the authors and do not necessarily represent those of their affiliated organizations, or those of the publisher, the editors and the reviewers. Any product that may be evaluated in this article, or claim that may be made by its manufacturer, is not guaranteed or endorsed by the publisher.

Copyright $\odot 2021$ Wang, Wang, Han, Sun, Feng, Ye and Zhang. This is an openaccess article distributed under the terms of the Creative Commons Attribution License (CC BY). The use, distribution or reproduction in other forums is permitted, provided the original author (s) and the copyright owner(s) are credited and that the original publication in this journal is cited, in accordance with accepted academic practice. No use, distribution or reproduction is permitted which does not comply with these terms. 